\title{
Herd immunity: evidence and way forward for COVID-19
}

Sampatha Goonewardena ${ }^{1}$, Indumini Gunatilake ${ }^{2}$, Bhumini Karunarathna ${ }^{3}$, Chithramali Rodrigo ${ }^{3}$, Dharshini Kantharuban ${ }^{4}$, Maheeka Seneviwickrama ${ }^{1}$, Pushpa Jayawardana, Ishanka Ayeshwari Talagala $^{3^{*}}$

${ }^{1}$ Department of Community Medicine, Faculty of Medical Sciences, University of Jayewardenepura, Sri Lanka; ${ }^{2}$ Postgraduate Institute of Medicine, University of Colombo, Sri Lanka; ${ }^{3}$ Ministry of Health, Sri Lanka; ${ }^{4}$ Provincial Director of Health Services Office, Eastern Province, Trincomalee, Sri Lanka; ${ }^{5}$ No affiliation

"Correspondence: drishanka@gmail.com https://orcid.org/0000-0001-6351-2079

DOI: https://doi.org/10.4038/jccpsl.v26i5.8348

Received on 17 May 2020

Accepted on 25 May 2020

\section{Highlights}

- Lancet reported that with an $\mathrm{R}_{0}$ of roughly 2.2 for SARS-Cov-2, it would require a herd immunity of $60 \%$ in the population. However, if many people are exposed to the virus, they will develop antibodies and will have natural immunity.

- The only mechanism to provide immediate protection from the disease as well as achieve herd immunity rapidly is through mass vaccination.

\section{What is herd immunity?}

Herd immunity is an epidemiological concept on the immune status of a population. The Centers for Disease Control and Prevention (CDC) defines herd immunity as a situation in which a sufficient proportion of the population is immune to the infectious disease (either through vaccination or prior illness), making its spread from one person to another person highly unlikely. In other words, the presence of immune individuals protects nonimmune people from the infection (1). If there is no herd immunity, people will continue to contract the disease and spread it to others.
Herd immunity, also known as community immunity, can be passed from person to person via two methods; firstly by means of vaccination, where the individuals develop immunity to the disease, so that the infectious agent could be neutralized when they come in contact, making it much less likely to pass on to others. Herd immunity therefore is an indirect natural measure and does not give high levels of individual protection unlike vaccination. Thus, herd immunity is more effective as the percentage of people vaccinated increases (2). However, if many people are exposed to the virus, they will develop antibodies and will have natural immunity as well (3). Secondly, if nearly everyone is immune, then 
almost no one will spread the disease. Therefore, even people who have not been vaccinated can be shielded by herd immunity, because vaccinated people around them are not getting sick.

However, with regard to COVID-19, the importance of herd immunity as a long term control strategy is unclear due to not knowing how long the immunity against the virus, SARS-CoV-2 would last and its behaviour in the community (4).

It is said that mass vaccination is practised not only to protect the individuals themselves from mild infectious diseases, but also to safeguard those who are vulnerable and ineligible for vaccination. For example, Rubella vaccine causes only a mild infection in childhood, but it prevents pregnant mothers who are not immune from delivering newborns with congenital rubella syndrome. However, the concept that vaccine-based herd immunity is immediately attainable has been disproved in the United States in relation to the measles vaccine, as mass vaccination of the population in the 1960 s failed to prevent outbreaks of measles in the country (5).

With regard to a second infection, it is unknown whether a person can get infected for the second time, but it is unlikely to get infected in the short term. Evidence from other coronaviruses suggests that a person would have at least some immunity for a few years (6). It is estimated that with a pronounced $\mathrm{R}_{0}$, which represents the number of new infections estimated to stem from a single case, of about 2.2 for SARS-Cov-2, it would require a herd immunity of $60 \%$ in the population (7). Development of antibodies following SARS-CoV-2 virus infection is known to provide some protection. However, whether it could protect a person against reinfection is of much concern. Thus, more scientific evidence is needed to prove these (8).

\section{Public health implications}

There are two ways in which herd immunity could be established, namely, through exposure to those with the disease or vaccination. Coronavirus disease 2019 is a newly emerged disease. Therefore, the possibility of reaching the required threshold of herd immunity through this natural protection mechanism is beyond reach. Further, since this is a new disease and that no one is immune to it yet, it can lead to a fair proportion of people who are likely to develop complications leading to severe infection and death (3). In fact, the Government of UK had plans initially, to let the infection spread among the nation, in order to achieve herd immunity with the intention of containing the infection. Contrary to the negative impressions expressed on herd immunity related to COVID-19, some scientists are of the opinion that the infection is not likely to end but become an endemic disease where herd immunity would then have to play a major role. It is also stated that herd immunity would have been a possibility, if an uncontrolled epidemic was allowed to take place. However, it is likely to have overwhelmed the healthcare services leading to severe complications and further increase in the death rate. The violation of human rights at the expense of herd immunity, the attributes of which are still not understood, is another debatable matter. The only mechanism to provide immediate protection from the disease as well as achieve herd immunity rapidly is through mass vaccination. However, the development of an effective vaccine is not foreseeable in the near future. Good news is that several research projects have been embarked upon all over the world in search of an effective vaccine. Also, another implication of the COVID-19 is that, since it is new no one knows how protective the immune response is and the long-term effects of it (3).

It is of utmost importance to know how the host reacts to this new virus, immunity level after infection, whether it is lifelong or not, to plan the treatment options. The availability of a new drug or an already existing drug to use for this purpose is also important, so that the population can be treated easily. This will in turn reduce the mortality rate. The availability of a prophylactic drug will reduce the morbidity, and thereby reduce the caseload and prevention made easy. However, it is a relatively new virus which is not well researched, and therefore researchers are not fully aware how protective the antibody response is going to be over long term (3). 


\section{Gaps in the existing knowledge}

Since the virus is less than six months old, a vast knowledge gap is inevitable. All over the world, research is being carried out with available data to know about the virus genome, its strain, virility, pathogenicity, treatment options, possibility of developing a vaccine and availability of a potent vaccine against this new virus. Clinical trials are undertaken but published data are not yet available. Several ongoing research on antivirals, other medications and vaccines are in the pipeline. Still scientists have no answers to whether an individual will develop a second infection, whether the second infection will lead to more complications and why some get mild symptoms while others get severe infections (9).

\section{Applicability of the evidence to Sri Lanka}

The use of hydroxychloroquine (HCL) was recommended in a position paper initiated by the GMOA. Hydroxychloroquine is readily available in Sri Lanka as this was used for treatment of Malaria. This is currently under investigation in clinical trials for pre-exposure or post-exposure prophylaxis of SARS-CoV-2 infection, and treatment of patients with mild, moderate, and severe COVID-19. If this trial becomes a success, Sri Lanka can initiate treatment with HCL.

The Indian Council for Medical Research has recommended chemoprophylaxis with HCL for asymptomatic healthcare workers who treat patients with suspected or confirmed COVID-19 and asymptomatic household contacts of confirmed cases. Since there are no peer reviewed publications that have evaluated either drug for exposure prophylaxis of SARS-Cov-2 infection, it is used with caution. The authors reported that every patient is well-informed of its use since its efficacy and potential risks are still to be documented. All outcome events are documented for further reference (10).

French authors conducted a single arm protocol using HCL and azithromycin therapy. Although the sample size was small, results showed that HCL treatment was significantly associated with viral load reduction in COVID-19. Although the results are promising, the authors recommend more studies using randomized clinical trials with larger sample sizes (11).

Ferner and Aronson recently reported that although there are several trials conducted on the efficacy of HCL, properly conducted randomized controlled trials are needed to prove its efficacy (12). Investigators from Harvard Medical School and Massachusetts General Hospital after conducting extensive reviews stated that although HCL is effective against malaria, rheumatoid arthritis and Lupus, it is unlikely to be effective for COVID-19 infection (13). Research on immunity level and treatment options is still on-going and evidencebased results from randomized clinical trials are much needed.

\section{Author Declaration}

Author contributions: SG and IG have made substantial contributions to the conception, drafting and critical analysis for the intellectual content of the manuscript. All authors agree to be accountable for all aspects of the work in ensuring that questions related to the accuracy or integrity of any part of the work are appropriately investigated and resolved. All authors have read and approved the manuscript.

\section{References}

1. Gillespie C. Can herd immunity help stop coronavirus? What we know now. Explore Health, 18 March 2020. Available from: https:// www.health.com/condition/infectious-diseases/ coronavirus/herd-immunity.

2. Thoughtscapism. The simple math of herdimmunity. Thoughtscapism, 20 April 2015. Available from: https://thoughts capism.com /2015/04/20/the-simple-math-of-herd-immunity/. Accessed 20 April 2015.

3. Barr S. Coronavirus: what is herd immunity and is it a possibility for the UK? Independent, 15 April 2020. Available from: https:// www. independent. co.uk/life-style/health-and-families/coronavirusherd-immunity-meaning-definition-what-vaccineimmune-covid-19-a9397871.html. 
4. Yong E. The UK's coronavirus 'herd immunity' debacle. The Atlantic, 16 March 2020. Available from: https://www.theat lantic.com/ health/archive/2020/03/coronavirus-pandemicherd-immunity-uk-boris-johnson/608065/.

5. Obukhanych T. Herd immunity: can mass vaccination achieve it? Available from: https:// www.tetyanaobukhanych.com/herd_immunity.ht $\mathrm{ml}$.

6. Berezow A. Coronavirus: what will it take to achieve COVID-19 herd immunity? American Council on Science and Health, 29 April 2020. Available from:https://www.acsh.org/ news/2020/04/29/coronavirus-what-will-it-takeachieve-covid-19-herd-immunity-14752.

7. Altmann DM, Douek DC, Boyton RJ. What policy makers need to know about COVID-19 protective immunity. Lancet 2020; 395: 1527-29. DOI:10.1016/S0140-6736(20)30985-5.

8. McKenna S. What immunity to COVID 19 might actually mean? Scientific American, 10 April 2020. Available from: https://www.scientifi camerican.com/article/what-immunity-to-covid19-really-means.
9. Iftikhar N. Herd immunity: what it means for COVID 19? Healthline, 2 April 2020. Available from: https://www.healthline .com/health/herdimmunity.

10. Rathi S, Ish P, Kalantri A, Kalantri S. Hydroxychloroquine prophylaxis for COVID -19 contacts in India. Lancet Infectious Diseases 17 April 2020. http:// oi. org/10.1016/S1473-3099(20) 30313-3.

11. Gautret P, Lagier JC, Parola P, et al. Hydroxy chloroquine and azithromycin as a treatment of COVID-19: results of an open-label nonrandomized clinical trial. International Journal of Antimicrobial Agents 2020; 56(1): 105949. DOI: 10.1016/j. ijantimicag. 2020.105949.

12. Ferner RE, Aronson JK. Chloroquine and hydroxychloroquine in COVID-19: Use of these drugs is premature and potentially harmful. $B M J$ 2020; 369: m1432. DOI: 10.1136/bmj.m1432.

13. Lutz R. Review: chloroquine, hydroxy chloroquine likely ineffective for COVID-19. Contagion Live, 30 April 2020. Available from: https:// www.contagionliv.com/news/chloroqu inehydroxychloroquine-ineffective-covid-19. 